\title{
Evaluation of Predisposing Metabolic Risk Factors for Portopulmonary Hypertension in Patients with NASH Cirrhosis
}

\author{
Fatih Türker $\mathbb{D}^{\prime}$, Tolga Sahın ${ }^{2}$, Alihan Oral ${ }^{2}$, Erdem Koçak ${ }^{3}$, Betül Çavușoğlu Türker ${ }^{4}$, \\ Adil Niğdelioğlu², Hayriye Esra Ataoğlu' \\ 'University of Health Sciences, Haseki Health Training and Research Hospital, Internal Medicine Clinic, İstanbul, Turkey; ${ }^{2}$ Demiroglu Bilim University, \\ Florence Nightingale Hospital Internal Medicine Clinic, İstanbl, Turkey; ${ }^{3}$ Istinye University, Liv Hospital Vadi Istanbul, Gastroenterology, İstanbul, \\ Turkey; ${ }^{4}$ University of Health Sciences, Taksim Health Training and Research Hospital, Internal Medicine Clinic, İstanbul, Turkey \\ Correspondence: Fatih Türker, Aksaray, Dr. Adnan Adıvar Cd, No: 9, İstanbul, Tel +090536472I 656, Fax +02I2 453 20 00, Email fatihturker I985@hotmail.com
}

Purpose: Metabolic parameters are important for the development of portopulmonary hypertension (PoPH) during nonalcoholic steatohepatitis (NASH)-associated cirrhosis. This study evaluated patients with NASH-associated cirrhosis to determine metabolic risk factors for portopulmonary hypertension.

Patients and Methods: Data on 171 patients (120 men and 51 women) with NASH-associated cirrhosis who were seen in Florence Nightingale Hospital's gastroenterology Clinic from 2009 to 2018 was obtained from the Hospital database. A pulmonary artery systolic pressure $>35 \mathrm{mmHg}$ was defined as $\mathrm{PH}$ (pulmonary hypertension) according to standard transthoracic echocardiography. Portal hypertension was diagnosed from clinical symptoms and dilated portal veins shown by abdominal ultrasound or computed tomography (CT). Pulmonary patients with portal hypertension were diagnosed with portopulmonary hypertension (PoPH).

Results: A total of 171 patients with NASH-associated cirrhosis were included in this study. Of these, 43 patients had PoPH. These patients had increased TSH $(p=0.004)$, bilirubin $(p=0.023)$ and triglyceride $(p=0.048)$ levels, higher MELD scores $(p=0.018)$ and decreased hemoglobin $(\mathrm{p}=0.05)$. MELD score and hemoglobin, total bilirubin, TSH, and triglyceride levels were all included in a multivariate logistic regression model and TSH levels were independently associated with increased risk of PoPH.

Conclusion: Increased TSH is an independent risk factor for PoPH.

Keywords: pulmonary hypertension, portopulmonary hypertension, NASH cirrhosis

\section{Introduction}

Portopulmonary hypertension (PoPH) is defined as pulmonary arterial hypertension (PAH) associated with cirrhotic or noncirrhotic portal hypertension. ${ }^{1}$ Pulmonary hypertension is classified into five groups, and portopulmonary hypertension $(\mathrm{PoPH})$ is included in the first group because of hemodynamic similarities to other causes of precapillary pulmonary hypertension. Thus, transthoracic echocardiography (TTE) should be performed to screen cirrhotic patients for systolic pulmonary arterial pressure (sPAP). TTE is a reliable screening tool to estimate cardiac function. Systolic pulmonary arterial pressure is calculated by measuring the velocity of the tricuspid regurgitation jet to estimate the tricuspid pressure gradient during systole and calculating pressure using the modified Bernoulli equation, to which an estimate of right atrial pressure is added.

A strong correlation between sPAP and PAP obtained by right-heart catheterization has been shown in the general population. ${ }^{2}$ A pulmonary artery systolic pressure $>35 \mathrm{mmHg}$ was defined as pulmonary hypertension $(\mathrm{PH})$ using standard echocardiography. ${ }^{3} \mathrm{PH}$ is confirmed when right heart catheterization demonstrates that pulmonary artery pressure $(\mathrm{mPAP}) \geq 25 \mathrm{mmHg}$ at rest with a normal pulmonary artery wedge pressure $\leq 15 \mathrm{mmHg}$. ${ }^{4}$ Right-heart catheterization is the gold standard for a "definitive diagnosis" of $\mathrm{PH}$. While the mechanism of PoPH is similar to 
other forms of pulmonary arterial hypertension (PAH), the exact pathophysiology remains unclear and the prevalence of PoPH has ranged from $0.5 \%$ and $5 \%$ among patients with portal hypertension in prior studies. ${ }^{5-7}$

Nonalcoholic fatty liver disease (NAFLD) is defined as the presence of chronic hepatic steatosis and inflammation in the absence of excessive alcohol consumption and is associated with metabolic comorbidities, such as obesity, diabetes mellitus, and dyslipidemia. ${ }^{8,9}$ NAFLD progresses to non-alcoholic steatohepatitis (NASH) and has the potential to progress further into cirrhosis. Several studies have shown that the prevalence of thyroid dysfunction is significantly higher in patients with NAFLD. ${ }^{10}$ This may be explained by the strong association between thyroid hormone levels and metabolic syndrome as well as the disturbance of lipid metabolism. ${ }^{11}$ However, few studies have characterized predisposing metabolic risk factors including thyroid hormone levels in cirrhotic patients with PoPH. The aim of the current study was to evaluate the association between PoPH and risk factors in patients with NASH-associated cirrhosis.

\section{Materials and Methods}

\section{Study Participants}

This study was approved by the Ethics Committee of Florence Nightingale Hospital, University of Bilim, Istanbul, Turkey, and the clinical research ethics committee at Florence Nightingale Hospital (approval number 2019-16-03; approved on 8 June 2019) and performed in accordance with National Institute of Health guidelines. The study was conducted in accordance with the principles of good clinical practice and the Declaration of Helsinki. All data were collected anonymously without including patient-identifying information, and consent to review patient medical records was not required by the Ethics Committee of Florence Nightingale Hospital. Patients $>18$ years of age with cirrhosis resulting from nonalcoholic steatohepatitis (NASH) who had pulmonary artery systolic pressure $>35 \mathrm{mmHg}$ measured by TTE were included in the study. Patients $\leq 18$ years, with cardiovascular disease (congenital heart disease, valvular heart disease, or coronary heart disease), severe lung disease, thromboembolism, kidney disease, or active infection were excluded.

Patients with NASH-associated cirrhosis were Child B and Child C patients from whom a biopsy was contraindicated. Those with NASH-associated liver cirrhosis who did not have excessive alcohol intake (considered as an average daily consumption of alcohol $>30 \mathrm{~g} /$ day in men and $>20 \mathrm{~g} /$ day in women), negative test results for hepatitis B surface antigen and hepatitis $\mathrm{C}$ virus antibody, and no drug treatments known to cause liver steatosis, such as corticosteroids and estrogens, were included. In total, 171 patients (120 men and 51 women) with NASH-associated cirrhosis who were seen in Florence Nightingale Hospital's gastroenterology clinic from 2009 to 2018 were included in the study. Ultrasonography and CT were performed on all NASH patients. A pulmonary artery systolic pressure $>35 \mathrm{mmHg}$ was defined as PH using standard TTE. Data for the study analyses was obtained from dispensing records and profile data in the electronic hospital management system at Florence Nightingale Hospital. Patient demographic information, medical history, TTE, abdominal ultrasound, and laboratory examination data were obtained through standardized data collection. Demographic data, medical history, vital signs at admission, medications, and final diagnosis were obtained from electronic patient medical records.

\section{Laboratory Measurements}

Routine blood samples were drawn between 6 and 7 am after a 12-hour fast and immediately analyzed. Liver and kidney function, blood glucose, serum lipid, hemogram, and inflammatory markers like sedimentation and C-reactive protein (CRP) were measured in the Department of Laboratory Medicine at Florence Nightingale Hospital, University of Bilim. Laboratory data were obtained from the hospital's electronic patient medical records. Biochemical parameters were measured for all participants.

SPAP was calculated by measuring the velocity of the tricuspid regurgitation jet to estimate the tricuspid pressure gradient during systole and calculating pressure using the modified Bernoulli equation, to which an estimate of right atrial pressure is added.

Portal hypertension was diagnosed using clinical symptoms (ascites, history of gastrointestinal hemorrhage, abdominal wall varicose veins, and splenomegaly) and expanded portal veins were determined by abdominal ultrasound or CT. 
A pulmonary artery systolic pressure $>35 \mathrm{mmHg}$ was defined as pulmonary hypertension ( $\mathrm{PH}$ ). $\mathrm{PH}$ patients with portal hypertension were diagnosed with PoPH. Based on diagnosis, patients were divided into those with or without portopulmonary hypertension. Biochemical and metabolic parameters were compared between the two groups.

\section{Statistical Analysis}

Data are expressed as the mean \pm standard deviation and statistical analysis was performed using SPSS 24.0 (SPSS Inc., Chicago, IL, USA). Basic descriptive statistics were measured including the means, standard deviations, ranges, and percentages. The normality of the distribution was examined using the Kolmogorov-Smirnov test. Mean values between two independent groups were compared using the Mann-Whitney $U$-test for continuous variables and the $\chi^{2}$ test for categorical parameters and comparisons between three or more subgroups were performed using ANOVA and KruskalWallis $h$-tests. Bivariate correlations were assessed by Pearson's (continuous variables). Differences were considered statistically significant if the two-tailed $\mathrm{P}$ value was $<0.05$.

\section{Results}

In total, 171 patients with NASH-associated cirrhosis were included in this study, of whom $29 \%$ were female, and the median age of the patients was 55.9 years. Most patients $(\mathrm{N}=155)$ were complicated with portal hypertension and 46 and 43 patients had $\mathrm{PH}$ and $\mathrm{PoPH}$, respectively. The patient demographics and biochemical parameters are shown in Table 1. Higher body mass index was associated with PoPH $(\mathrm{p}=0.005)$ and there was no significant difference in CHILD PUGH scores $(\mathrm{p}=0.344)$ or albumin, liver, and renal function test results between patients with and without PoPH (Table 1). PoPH patients had a higher MELD score $(\mathrm{p}=0.018)$, higher total bilirubin $(\mathrm{p}=0.023)$ and triglyceride $(\mathrm{p}=0.048)$ levels, and lower hemoglobin $(\mathrm{p}=0.05)$ than patients without PoPH. Patients with PoPH were significantly older than those without. There was no significant difference between cardiac function and ejection fraction (EF) $(59,7 \pm 3,758.8 \pm 4,8$ $\mathrm{p}=0.174$ ), or levels of tumor markers (eg, AFP, CEA, CA19-9 and PoPH) between the groups (Table 1). Higher TSH was associated with an increased risk of PoPH. However, there was no significant difference in FreeT3 and FreeT4 levels between patients with and without PoPH.

Complications associated with cirrhosis in patients with and without PoPH are shown in Table 2. There was no significant difference in portal vein thrombosis, esophageal varices, hepatic encephalopathy, or spontaneous bacterial peritonitis between the two groups. Ascites was more common in patients with PoPH ( $\mathrm{p}=0.008)$. Age, MELD score, and $\mathrm{Hb}$, total bilirubin, TSH, ascites, and triglyceride levels were included in a multivariate logistic regression model, and only increased TSH level was independently associated with an increased risk of PoPH (Table 3).

\section{Discussion}

This study investigated the risk factors of PoPH in patients with NASH-associated cirrhosis. A total of 171 patients were included in the study, 43 of whom were diagnosed with PoPH. Patients with PoPH exhibited higher levels of TSH, total bilirubin, and triglycerides, higher MELD scores, higher incidence of ascites, and lower levels of hemoglobin than those without. All these parameters were included in a multivariant logistic regression model and higher TSH levels were independently associated with an increased risk of PoPH. Few studies have measured an association between patients with cirrhosis and PoPH. Li et al ${ }^{12}$ investigated the prognosis and prevalence of $\mathrm{PoPH}$ in liver transplantation recipients in China and demonstrated that low hemoglobin level was an independent risk factor for PoPH and screening for PoPH was essential for patients with advanced liver disease. The current study found an association between TSH level and PoPH in patients with cirrhosis that was not seen previously.

PoPH occurs in $7-10 \%$ of patients with PAH. Symptoms of PoAH are generally non-specific, and patients are often asymptomatic at the time of diagnosis. ${ }^{13-15}$ Studies have indicated that right heart dysfunction progresses with the development of $\mathrm{PoPH}$ and aggravates congestion as liver disease worsens. ${ }^{15}$ Of the laboratory parameters examined in this study, patients with PoPH had higher hemoglobin levels, MELD scores, and total bilirubin than patients without $\mathrm{PoPH}$, reflecting more serious liver disease.

TTE is a reliable screening tool to estimate cardiac function. Pulmonary artery systolic pressure $>35 \mathrm{mmHg}$ was defined as pulmonary hypertension $(\mathrm{PH})$ using standard echocardiography. $\mathrm{PH}$ is confirmed when right heart 
Table I Demographics and Biochemical Parameters of the Study Patients

\begin{tabular}{|c|c|c|c|}
\hline Variables & PoPH & nonPoPH & $P$ value \\
\hline Age & $58 \pm 8$ & $53 \pm 11$ & $0.033 *$ \\
\hline Gender M/F & $27 / 16$ & $93 / 35$ & 0.221 \\
\hline MELD score & $17.5 \pm 5.1$ & $15.6 \pm 4.3$ & $0.018 *$ \\
\hline CHİLD PUGH score & $8.8 \pm 1.9$ & $8.5 \pm 1.9$ & 0.344 \\
\hline BMI & $29.7 \pm 5.6$ & $27.9 \pm 5.2$ & $0.05 *$ \\
\hline Hemoglobin $(g / d l)$ & $10.8 \pm 1.5$ & $11.4 \pm 1.9$ & $0.05 *$ \\
\hline Platelet $\left(10^{3} / \mu \mathrm{L}\right)$ & $92,116 \pm 64,305$ & $94,378 \pm 64,305$ & 0.911 \\
\hline INR & $1.7 \pm 0.3$ & $1.6 \pm 0.4$ & 0.958 \\
\hline Fasting Glucose (mg/dl) & $111 \pm 34$ & $120 \pm 46$ & 0.219 \\
\hline Creatinine (mg/dl) & $1.1 \pm 0.5$ & $0.9 \pm 0.5$ & 0.053 \\
\hline Bilirubin (mg/dl) & $4.8 \pm 6.8$ & $3.8 \pm 4.6$ & 0.023 \\
\hline AST (U/L) & $61.8 \pm 50.6$ & $6 I . I \pm 56.8$ & 0.922 \\
\hline ALT (U/L) & $39.1 \pm 33.8$ & $39.4 \pm 34.7$ & 0.621 \\
\hline Albumin $(g / d L)$ & $2.8 \pm 0.5$ & $3.0 \pm 0.5$ & 0.138 \\
\hline TSH (mlU/L) & $3.5 \pm 3.32$ & $2.3 \pm 1.75$ & $0.004 *$ \\
\hline Free T3 (mlU/L) & $3.1 \pm 0.9$ & $3.2 \pm 0.8$ & 0.525 \\
\hline Free T4 (mlU/L) & $13.2 \pm 7.6$ & $12.3 \pm 6.7$ & 0.486 \\
\hline Cholesterol (mg/dl) & $119 \pm 40$ & $130 \pm 42$ & 0.253 \\
\hline Triglyceride (mg/dl) & $03.1 \pm 104.6$ & $81.6 \pm 34.4$ & $0.048 *$ \\
\hline AFP & $4.8 \pm 12.5$ & $4.56 \pm 8.78$ & 0.897 \\
\hline CEA & $3.69 \pm 2.48$ & $3.58 \pm 1.79$ & 0.764 \\
\hline CA19-9 & $57.2 \pm 90.1$ & $38.82 \pm 46.4$ & 0.112 \\
\hline
\end{tabular}

Note: *Statistically significant variables $(p<0.05)$.

Abbreviations: PoPH, portopulmonary hypertension; nonPoPH, nonportopulmonary hypertension; BMI, body mass index; AFP, alphafetoprotein; CEA, carcinoembryonic Antigen; AST, aspartate aminotransferase; ALT, alanine aminotransferase; TSH, thyroid stimulating hormone; INR, international normalized ratio.

Table 2 Association Between Complications of Cirrhosis and Portopulmonary Hypertension

\begin{tabular}{|l|l|l|l|}
\hline Variables & PoPH (n:43) & nonPoPH (n: I 29) & P value \\
\hline Spontan Bacterial Peritonitis & $3(\% 8.1)$ & $10(\% 8.9)$ & 0.878 \\
\hline Hepatic Encephalopathy & $19(\% 47.5)$ & $72(\% 58.1)$ & 0.242 \\
\hline Esophageal Varices & $16(\% 39)$ & $44(\% 35.2)$ & 0.658 \\
\hline Ascites & $42(\% 97.7)$ & $104(\% 81.3)$ & $\mathbf{0 . 0 0 8}$ \\
\hline Portal Vein Thrombosis & $5(\% 11.6)$ & $6(\% 4.7)$ & 0.109 \\
\hline
\end{tabular}

Note: *Statistically significant variables $(p<0.05)$.

Abbreviations: PoPH, portopulmonary hypertension; nonPoPH, nonportopulmonary hypertension. 
Table 3 Results of Multivariate Logistic Regression Analysis

\begin{tabular}{|l|l|l|l|}
\hline Variable & $\mathbf{P}$ & OR & $\% 95 \mathbf{~ C l}$ \\
\hline Age & 0.194 & 0.030 & $0.985-1.078$ \\
\hline TSH $(\mathrm{mlU} / \mathrm{L})$ & $0.041^{*}$ & 0.181 & $1.007-1.428$ \\
\hline Bilirubin $(\mathrm{mg} / \mathrm{dl})$ & 0.709 & -0.019 & $0.886-1.086$ \\
\hline MELD & 0.137 & 0.093 & $0.971-1.240$ \\
\hline BMI & 0.448 & 0.029 & $0.955-1.110$ \\
\hline Hemoglobin $(\mathrm{g} / \mathrm{dl})$ & 0.153 & -0.166 & $0.675-1.063$ \\
\hline Triglyceride $(\mathrm{mg} / \mathrm{dl})$ & 0.332 & 0.004 & $0.996-1.012$ \\
\hline
\end{tabular}

Note: *Statistically significant variables $(p<0.05)$.

Abbreviations: BMI, body mass index; TSH, thyroid stimulating hormone.

catheterization demonstrates that pulmonary artery pressure (mPAP) $\geq 25 \mathrm{mmHg}$ at rest with normal pulmonary artery wedge pressure $\leq 15 \mathrm{mmHg}$. Although right heart catheterization is recommended for the definitive diagnosis of pulmonary hypertension, many studies show that patients in this group can be diagnosed with echocardiography by using an sPAP value instead of invasive catheterization in order to avoid invasive interventions and associated complications such as bleeding, thromboembolism, and infection that may develop in chronic liver patients. Calle et al reported that the NPV and PPV of sPAP values $>30 \mathrm{~mm} / \mathrm{Hg}$ were $>55 \%$ and $100 \%$, respectively. ${ }^{16,17}$ Liberal et al reported that the sensitivity and specificity of $\mathrm{SPAP}$ values $>38 \mathrm{~mm} / \mathrm{Hg}$ were $100 \%$ and $>80 \%$, respectively. ${ }^{15}$ In the current study, patients with sPAP $>35 \mathrm{~mm} / \mathrm{Hg}$ values were enrolled from patient medical record data collected prospectively.

The thyroid is one of the most important organs responsible for regulating hemodynamics in the human body. The liver plays an important role in thyroid hormone metabolism by controlling peripheral deiodination of thyroid hormones, producing carrier proteins like thyroxine-binding globulin, transthyretin, and albumin and playing a role in the clearance of thyroid hormones. ${ }^{18}$ Some studies show that Free T3 (FT3) and Free T4 (FT4) levels are significantly lower and TSH levels are significantly higher in liver cirrhosis patients. ${ }^{19-24}$ An association between thyroid disorder and PH has been reported, ${ }^{25}$ but few studies have evaluated the association between thyroid function and PoPH. In the current study, patients with PoPH had higher TSH levels than those without, while Free T3 and Free T4 were in the normal range. Thus, these patients were characterized as having subclinical hypothyroidism. Hypothyroidism has an influence on the cardiovascular system as a result of increased systemic vascular resistance and diastolic blood pressure. ${ }^{26,27}$ An increase in cardiac afterload affects the renin-angiotensin axis and reduces cardiac output, leading to higher pressure in the pulmonary veins. Hypothyroidism can also increase the oxidation of LDL. ${ }^{28,29}$ Thus, the presence of oxidative-LDL and hypothyroidism can increase vascular systemic and pulmonary resistance. Findings from this study showed a significant difference in the average age of patients in the two groups. Elevated TSH, total bilirubin, and triglyceride levels in PoPH patients may represent the severity of liver disease progression with age. As a result, L-thyroxine treatment for TSH may have a beneficial effect on NASH and PoPH patients. L-thyroxine treatment could reorganize the hemodynamics of the human body and reduce complications in patients with NASH cirrhosis. Findings from this study also showed that elevated TSH was an independent risk factor for PoPH irrespective of these cardiac and vascular alterations. Thus, TSH and thyroid hormone levels should be routinely monitored, especially in patients with NAFLD and cirrhosis.

This study also found that high BMI and triglyceride levels were associated with PoPH. Increased body weight can increase systemic vascular inflammation, ${ }^{30,31}$ which can, in turn, cause angioproliferative alterations in the pulmonary vascular bed and pulmonary hypertension. 
This study has some limitations. It is a retrospective study and pulmonary hypertension cannot be confirmed with right heart catheterization even though RHC is the gold standard for diagnosing PoPH. PH was diagnosed using standard echocardiography.

\section{Conclusion}

Elevated TSH, total bilirubin and triglyceride levels, and decreased hemoglobin levels were strongly associated with the development of PoPH in patients with NASH-related cirrhosis. Moreover, elevated TSH was shown to be an independent risk factor for the development of PoPH. Future investigation should focus on the role of thyroid hormone in the development of PoPH in patients with NASH-related cirrhosis.

\section{Acknowledgments}

We would like to thank the editorial team and the participants who contributed to our study.

\section{Author Contributions}

All authors made a significant contribution to the work reported, whether that is in the conception, study design, execution, acquisition of data, analysis and interpretation, or in all these areas; took part in drafting, revising or critically reviewing the article; gave final approval of the version to be published; have agreed on the journal to which the article has been submitted; and agree to be accountable for all aspects of the work.

\section{Disclosure}

The author reports no conflicts of interest in this work.

\section{References}

1. Hervé P, Lebrec D, Brenot F, et al. Pulmonary vascular disorders in portal hypertension. Eur Respir J. 1998;11:1153-1166. doi:10.1183/ 09031936.98.11051153

2. Steckelberg RC, Tseng AS, Nishimura R, et al. Derivation of mean pulmonary artery pressure from noninvasive parameters. $J$ Am Soc Echocardiogr. 2013;26:464-468. doi:10.1016/j.echo.2013.01.006

3. Fisher MR, Forfia PR, Chamera E, et al. Accuracy of Doppler echocardiography in the hemodynamic assessment of pulmonary hypertension. $\mathrm{Am}$ J Respir Crit Care Med. 2009;179:615-621. doi:10.1164/rccm.200811-16910C

4. Simonneau G, Gatzoulis MA, Adatia I, et al. Updated clinical classification of pulmonary hypertension. J Am Coll Cardiol. 2014;63:746-753.

5. Chabot F, Gomez E, Boyer L, et al. Porto-pulmonary hypertension. Rev Mal Respir. 2006;23:629-641. doi:10.1016/S0761-8425(06)72078-2

6. Pilatis ND, Jacobs LE, Rerkpattanapipat P, et al. Clinical predictors of pulmonary hypertension in patients undergoing liver transplant evaluation. Liver Transplant off Publ Am Assoc Study Liver Dis Int Liver Transplant Soc. 2000;6:85-91.

7. Krowka MJ, Swanson KL, Frantz RP, McGoon MD, Wiesner RH. Portopulmonary hypertension: results from a 10-year screening algorithm. Hepatol Baltim Md. 2006;44(6):1502-1510. doi:10.1002/hep.21431

8. Puchakayala BK, Verma S, Kanwar P, et al. Histopathological differences utilizing the nonalcoholic fatty liver disease activity score criteria in diabetic (type 2 diabetes mellitus) and non-diabetic patients with nonalcoholic fatty liver disease. World J Hepatol. 2015;7:2610-2618. doi:10.4254/ wjh.v7.i25.2610

9. Sanyal AJ, Chalasani N. Trials and tribulations in drug development for nonalcoholic steatohepatitis. Clin Gastroenterol Hepatol. 2014;12:2104-2105. doi:10.1016/j.cgh.2014.08.005

10. Eshraghian A, Hamidian Jahromi A. Non-alcoholic fatty liver disease and thyroid dysfunction: a systematic review. World J Gastroenterol. 2014;20 (25):8102-8109. doi:10.3748/wjg.v20.i25.8102

11. Amarapurkar D, Kamani P, Patel N, et al. Prevalence of non-alcoholic fatty liver disease: population based study. Ann Hepatol. 2007;6(3):161-163. doi:10.1016/S1665-2681(19)31922-2

12. Li J, Zhuang O, Zhang X, et al.Prevalence and prognosis of portopulmonary hypertension in 223 liver transplant recipients. Can Respir J. 2018;2018:9629570. doi:10.1155/2018/9629570

13. Reymond M, Barbier L, Salame E, et al. Does portopulmonary hypertension impede liver transplantation in cirrhotic patients? A French multicentric retrospective study. Transplantation. 2018;102:616-622. doi:10.1097/TP.0000000000001981

14. Krowka MJ, Miller DP, Barst RJ, et al. Portopulmonary hypertension: a report from the US-based REVEAL registry. Chest. 2012;141:906-915.

15. Liberal R, Grant CR, Baptista R, et al. Porto-pulmonary hypertension: a comprehensive review. Clin Res Hepatol Gastroenterol. 2015;39 (2):157-167.

16. Colle IO, Moreau R, Godinho E, et al. Diagnosis of portopulmonary hypertension in candidates for liver transplantation: a prospective study. Hepatology. 2003;37:401-409. doi:10.1053/jhep.2003.50060

17. Raevens S, Colle I, Reyntjens K, et al. Echocardiography for the detection of portopulmonary hypertension in liver transplant candidates: an analysis of cutoff values. Liver Transpl. 2013;19:602-610. doi:10.1002/1t.23649

18. Malik R, Hodgson H. The relationship between the thyroid gland and the liver. $Q$ J Med. 2002;95:559-569. doi:10.1093/qjmed/95.9.559 
19. Saleem WM, Wadea FM. Evaluation of thyroid dysfunction in Egyptian chronic hepatitis c virus cirrhotic patients complicated with portal hypertension. Int J Sci Res. 2016;5:595-600.

20. El-Sawy AA, Tawfi KM. Low serum free and total tri-iodothyronine hormones as possible prognostic factors in liver cirrhotic patients because of chronic hepatitis C. Tanta Med J. 2015;43:46-51. doi:10.4103/1110-1415.158048

21. El-Feki MA, Abdalla NH, Atta MI, et al. Serum level of thyroid hormones in patients with chronic hepatitis C virus infection. Open J Endocr Metab Dis. 2016;6:126-134. doi:10.4236/ojemd.2016.63017

22. Antonelli A, Ferri C, Fallahi P, et al. Thyroid Involvement in patients with overt HCV-related mixed cryoglobulinaemia. QJM Int J Med. 2004;97 (8):499-506. doi:10.1093/qjmed/hch088

23. D'costa L, Dhume CY. Assessment of thyroid parameters in alcoholic liver disease. Int J Pharm Biosci. 2016;7:771-776.

24. Kayacetin E, Kisakol G, Kaya A. Low serum total thyroxine and free triiodothyronine in patients with hepatic encephalopathy due to non-alcoholic cirrhosis. Swiss Med Wkly. 2003;133:210-213.

25. Spencer CA, Takeuchi M, Kazarosyan M. Current status and performance goals for serum thyrotropin (TSH) assays. Clin Chem. 1996;42:140-145. doi:10.1093/clinchem/42.1.140

26. Vargas-Uricoechea H, Bonelo-Perdomo A, Sierra-Torres CH. Effects of thyroid hormones on the heart. Clin Investig Arterioscler. 2014;26:296-309. doi:10.1016/j.arteri.2014.07.003

27. Mohr-Kahaly S, Kahaly G, Meyer J. Cardiovascular effects of thyroid hormones. Z Kardiol. 1996;85:219-231.

28. Duntas LH, Mantzou E, Koutras DA. Circulating levels of oxidized low-density lipoprotein in overt and mild hypothyroidism. Thyroid. 2002;12:1003-1007. doi:10.1089/105072502320908349

29. Ittermann T, Baumeister SE, Völzke H, et al. Are serum TSH levels associated with oxidized low-density lipoprotein? Results from the Study of Health in Pomerania. Clin Endocrinol (Oxf). 2012;76:526-532. doi:10.1111/j.1365-2265.2011.04186.x

30. Siemińska L, Wojciechowska C, Walczak K, et al. Associations between metabolic syndrome, serum thyrotropin, and thyroid antibodies status in postmenopausal women, and the role of interleukin-6. Endokrynol Pol. 2015;66:394-403. doi:10.5603/EP.2015.0049

31. Tvarijonaviciute A, Jaillardon L, Cerón JJ, Siliart B. Effects of thyroxin therapy on different analytes related to obesity and inflammation in dogs with hypothyroidism. Vet J. 2013;196:71-75. doi:10.1016/j.tvj1.2012.08.005

International Journal of General Medicine

Dovepress

\section{Publish your work in this journal}

The International Journal of General Medicine is an international, peer-reviewed open-access journal that focuses on general and internal medicine, pathogenesis, epidemiology, diagnosis, monitoring and treatment protocols. The journal is characterized by the rapid reporting of reviews, original research and clinical studies across all disease areas. The manuscript management system is completely online and includes a very quick and fair peer-review system, which is all easy to use. Visit http://www.dovepress.com/testimonials.php to read real quotes from published authors.

Submit your manuscript here: https://www.dovepress.com/international-journal-of-general-medicine-journal 